\title{
The bHLH regulator pMesogenin1 is required for maturation and segmentation of paraxial mesoderm
}

\author{
Jeong Kyo Yoon and Barbara Wold ${ }^{1}$ \\ Division of Biology, California Institute of Technology, Pasadena, California 91125, USA
}

\begin{abstract}
Paraxial mesoderm in vertebrates gives rise to all trunk and limb skeletal muscles, the trunk skeleton, and portions of the trunk dermis and vasculature. We show here that germline deletion of mouse pMesogenin1, a bHLH class gene specifically expressed in developmentally immature unsegmented paraxial mesoderm, causes complete failure of somite formation and segmentation of the body trunk and tail. At the molecular level, the phenotype features dramatic loss of expression within the presomitic mesoderm of Notch/Delta pathway components and oscillating somitic clock genes that are thought to control segmentation and somitogenesis. Subsequent patterning and specification steps for paraxial mesoderm also fail, leading to a complete absence of all trunk paraxial mesoderm derivatives, which include skeletal muscle, vertebrae, and ribs. We infer that pMesogenin1 is an essential upstream regulator of trunk paraxial mesoderm development and segmentation.
\end{abstract}

[Key Words: bHLH; paraxial mesoderm; pMesogenin1; segmentation; somite; Notch]

Received September 12, 2000; revised version accepted November 2, 2000.

A defining feature of the vertebrate body plan is metameric segmentation of the musculoskeletal and neuromuscular systems. The origin of this basic anatomic plan during embryogenesis is segmentation of the paraxial mesoderm (Gossler and de Angelis 1998). Upon gastrulation, paraxial mesoderm cells segregate from axial and lateral mesoderm to form two identical strips of unsegmented tissue (presomitic mesoderm or PSM) on either side of the neural tube (Gossler and de Angelis 1998). This unsegmented tissue is converted, via a series of molecular and morphogenetic changes, into a string of tissue blocks called somites (Gossler and de Angelis 1998). Somitogenesis occurs sequentially in a strict head-to-tail progression along the body axis.

Although the underlying mechanism of segmentation remains unclear, it appears to be regulated by a molecular clock that is autonomous to paraxial mesoderm (Palmeirim et al. 1997; Jiang et al. 1998; Pourquie 1999; Dale and Pourquie 2000). Several regulatory components needed for segmentation have been identified recently, and a subset of these reveal the existence of the somitic clock by the dramatic oscillation of their RNA levels within the as yet unsegmented domain of the paraxial mesoderm or presomitic mesoderm (PSM) (Palmeirim et al. 1997; Forsberg et al. 1998; McGrew et al. 1998; Aulehla and Johnson 1999). Their periodicity of oscillation is directly correlated with the periodicity of somite

${ }^{1}$ Corresponding author.

E-MAIL Woldb@caltech.edu; FAX (626) 449-0756.

Article and publication are at www.genesdev.org/cgi/doi/10.1101/ gad. 850000 . formation. Murine and avian Lunatic fringe, murine HES1/avian cHairy, and murine HRT genes typify the oscillating transcript pattern in the PSM (Palmeirim et al. 1997; Forsberg et al. 1998; McGrew et al. 1998; Aulehla and Johnson 1999; Nakagawa et al. 1999; Jouve et al. 2000), and genetic assays in the Lunatic fringe null mutant mouse strains show that it is needed for correct segmentation (Evrard et al. 1998; Zhang and Gridley 1998). The Notch signaling apparatus is also intimately involved. Their RNAs do not oscillate in the PSM domain, but homozygous mutant mice for Notch1, Dll-1, DIl-3 (Pudgy) and RBP-Jk (Su [H]), all show defects in somite formation and segmentation in the mouse (Swiatek et al. 1994; Conlon et al. 1995; Oka et al. 1995; deAngelis et al. 1997; Kusumi et al. 1998). Similarly, disregulated expression and activity of Notch signaling components in Xenopus embryos generate defects in segmentation of paraxial mesoderm, even though the cellular events that execute somite formation in the frog differ from the epithelialization in mice and birds (Jen et al. 1997; Jen et al. 1999). Taken together, these data implicate the Notch system in regulating vertebrate somitogenesis and segmentation.

We reported previously the molecular cloning of the bHLH class gene pMesogenin 1 from mouse, together with its apparent ortholog from Xenopus (also called $\mathrm{Me}$ spo in Xenopus)(Joseph and Cassetta 1999; Yoon et al. 2000), and we showed that it is expressed specifically and prominently in paraxial mesoderm from gastrulation until overt somite formation. Together with two related mouse genes, MesP1 and MesP2, pMesogenin1 defines a 
novel subclass of bHLH proteins, whose members play significant roles in paraxial mesoderm development (Saga et al. 1996; Saga et al. 1997). MesP1/ 2 are expressed in the PSM in a spatially restricted, transient stripe located in the most rostral part of the PSM. The domain of MesP1/2 expression appears to prefigure a soon-to-form somite, and it is mutually exclusive relative to the domain of pMesogenin1 expression, which encompasses the entire caudal domain of the PSM (tailbud domain) (Yoon et al. 2000). Gain-of-function experiments in Xenopus embryos showed previously that ectopic $p M e$ sogenin1 can induce expression of functionally important ventrolateral mesodermal marker genes such as $X M y o D$ and $X w n t 8$, which encodes a signaling molecule capable of inducing a ventrolateral mesoderm identity in otherwise nonmesodermal cells of the animal cap (Yoon et al. 2000). Also induced by ectopic pMesogenin1 were ESR4 and ESR5, which are Hairy/Enhancer of Split related genes that are normally coexpressed with $p M e$ sogenin 1 in the unsegmented paraxial mesoderm of the frog (Jen et al. 1999; Yoon et al. 2000). Ectopic expression of pMesogenin 1 also suppressed expression of axial mesodermal (notochord) markers and, at high doses, disrupted normal notochord development (Yoon et al. 2000). These results strongly suggested that pMesogenin 1 plays a role in specification of all or part of the early paraxial mesoderm phenotype, presumably by regulating the transcription of downstream genes.

Whereas the Xenopus gain-of-function study (Yoon et al. 2000) suggested that pMesogenin1 is sufficient to in- duce multiple cellular and molecular features of the paraxial phenotype, it was not clear from that work whether pMesogenin 1 is essential for any or all aspects of paraxial mesoderm development. To find out if pMesogenin 1 is indeed necessary for aspects of paraxial mesoderm development, we disrupted the pMesogenin1 gene in the mouse germ line by homologous recombination in ES cells and construction of mice carrying the mutant alleles. The pMesogenin1 homozygous mutant embryos showed the most severe specific disruption of segmentation and maturation of posterior paraxial mesoderm of any gene we are aware of, whereas axial and lateral mesoderm developed quite normally.

\section{Results}

Morphological defects in the trunk and tail of pMesogenin 1 null animals

The mutant pMesogenin 1 allele (pMesogenin $\left.1^{\text {lac } Z \text {,neo }}\right)$ is a lacZ knock-in design in which the entire protein coding sequence of pMesogenin1 was replaced with a nuclear lacZ DNA sequence via in-frame fusion at the presumptive initiation codon of pMesogenin1 (Fig. 1A). $P G K-n e o$ sequence was subsequently removed from the locus by crossing with CMV-Cre transgenic mice, resulting in pMesogenin $1^{\text {lac } Z}$ allele.

Mice heterozygous for both pMesogenin1 mutant alleles in the129SvJ X C57BL6 hybrid background were fertile and morphologically normal. Both alleles produced

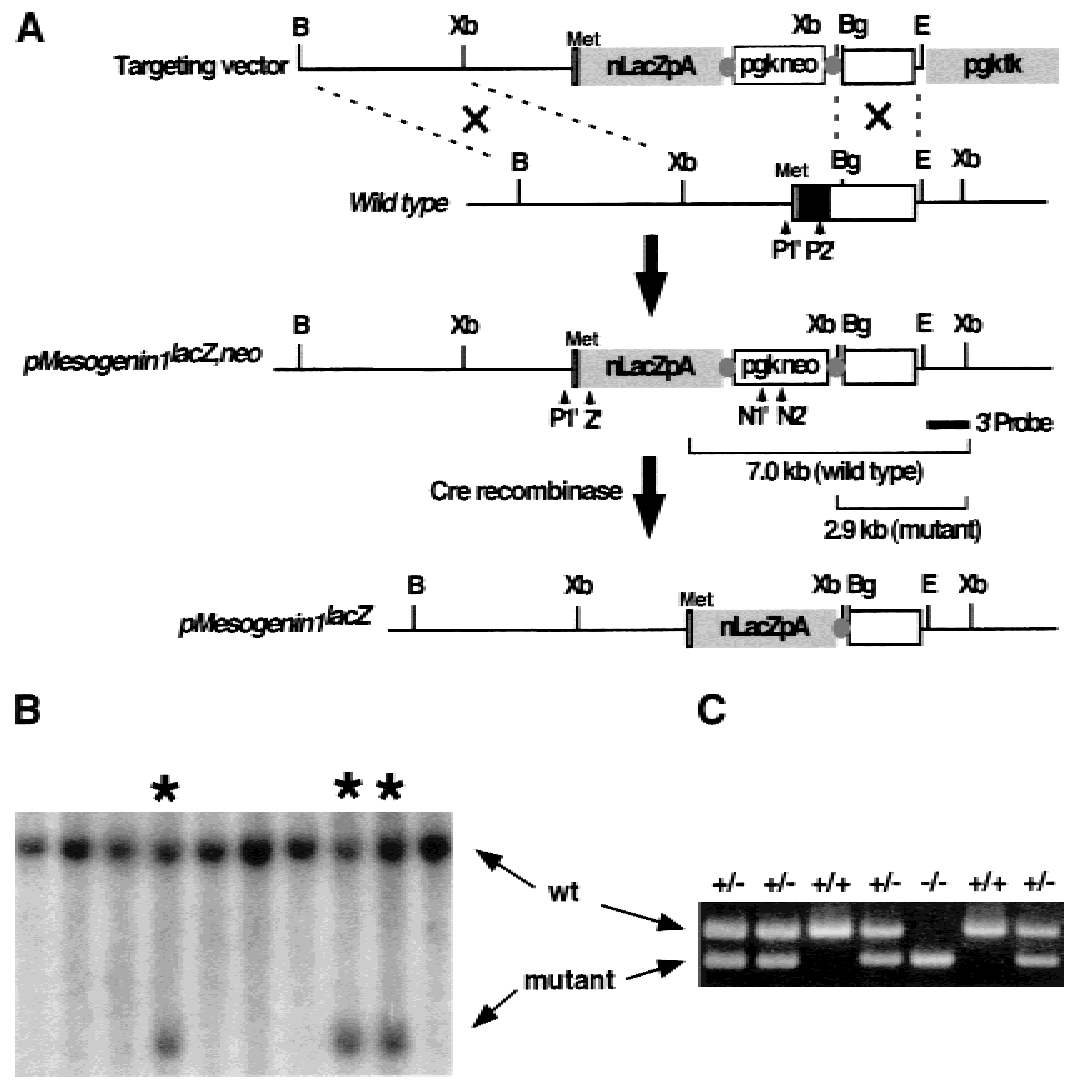

Figure 1. Targeted disruption of mouse $p M e$ sogenin 1 gene. $(A)$ In the targeting vector, the coding sequence of $p M e s o g e n i n 1$ gene was replaced with a nuclear $\beta$-galactosidase gene of E. coli in frame at the first methionine. A neomycin-resistance gene flanked by two loxP sites (Cre-recombinase recognition sites, gray colored circles), and thymidine kinase gene were used for positive and negative selections, respectively. Arrowheads indicate the locations of PCR primers. The bars below the mutated allele map indicate the expected band size of both wild type $(7 \mathrm{~kb})$ and mutated alleles $(2.9 \mathrm{~kb})$ in Southern blot analysis of XbaI-digested DNA. (B) Southern blot analysis of DNA isolated from transfected and selected ES (embryonic stem) cell clones. $(C)$ Genotype of embryos or fetuses are determined by PCR (polymerase chain reaction) using $\mathrm{P} 1, \mathrm{P} 2$, and $\mathrm{Z}$ primers as indicated in panel A. $344 \mathrm{bp}$ and $218 \mathrm{bp}$ PCR products are produced from wild type and the mutated alleles, respectively. The presence of the neo selection cassette after Cre recombination was monitored by PCR using N1 and N2 primers (see Materials and Methods for the primer sequences). 
identical phenotypes in homozygous animals, and the results presented here were collected from experiments using pMesogenin $1^{\text {lac } Z \text {, neo }}$ line. No homozygous animals were found among 32 newborn offspring from matings of pMesogenin $1^{\text {lacz,neo }}$ heterozygotes, suggesting that homozygous mutant animals die during gestation (Table 1). Embryos and fetuses were collected from additional heterozygote matings at different gestational timepoints. Genotypes followed Mendelian ratios until $10.5 \mathrm{dpc}$ (days postcoitum) (Table 1), after which the proportion of homozygous mutants began to decrease. Survival subsequently dropped throughout gestation.

Homozygous mutant embryos younger than $8.0 \mathrm{dpc}$ showed no detectable abnormalities in morphology. However, by 8.5 dpc a slightly expanded presomitic paraxial mesoderm domain was observed in homozygous null embryos (data not shown). More severe morphological abnormalities such as enlarged tailbud, kinked neural tube posterior to forelimb buds, and reduced tissue mass in the interlimb domain were evident at $9.0 \mathrm{dpc}$ (cf. Fig. $2 \mathrm{~A}$ to Fig. $2 \mathrm{~B}$ and $3 \mathrm{~A}$ ) and thereafter in homozygotes (Fig. 2C). Head and anterior body structures, including the developing heart, appeared normal. In the trunk posterior to the forelimbs, there were no identifiable somites or segment patterning, although the anterior-most somites (1-7) appeared normal, with decreases in somite size and disruption of myotomal patterning in somites $\sim 8-11$ (Figs. 2C, 4A,B). There were no detectable somites beyond somite 11. Ectopic pooled blood was observed in the enlarged tail bud of most, but not all, null embryos, and was also observed frequently in the trunk region of homozygous null embryos (asterisk in Fig. 2C). Transverse histological sections at the interlimb level of pMesogenin1 null embryos of 9.5 dpc showed virtually complete absence of paraxial mesoderm where somites would normally be and apparent expansion of vascular structures (Fig. 2F). Notochord and intermediate and lateral mesoderm all appeared grossly normal (Fig. 2F). In contrast, heterozygous littermates showed normal anatomic structures including the entire paraxial mesoderm and its major derivatives (Fig. 2E). Sections of trunk at the interlimb level of $11.5 \mathrm{dpc}$ null mutants showed even more pronounced defects (Fig. 2G,H): Myotomes were absent in homozygotes. Dorsal root ganglia (DRG), which normally mirror the segmentation pattern of somites, were present, indicating that neural crest cells emigrated and differentiated by the criteria of position and cell morphology, but the ganglia were not properly segmented (Fig. 2G,H). The established importance of signals from the somite for neural crest migration and

Table 1. Survival statistics for pMesogeninl mutants

\begin{tabular}{lccccc}
\hline & & & \multicolumn{2}{c}{ Genotypes (\%) } \\
\cline { 5 - 6 } Age of progeny & Total no. & $+/+$ & $+/-$ & $-/-$ \\
\hline $7.5-10.5 \mathrm{dpc}$ & 296 & 27.7 & 48.3 & 24.0 \\
$11.5-17.5 \mathrm{dpc}$ & 102 & 29.4 & 58.8 & 11.8 \\
Postnatal & 32 & 28.1 & 71.9 & 0 \\
\hline
\end{tabular}

patterning is consistent with this phenotype. Finally, the neural tube at the hindlimb level failed to close in homozygous mutants, although the rostral portion of neural tube closed normally (data not shown). Most lacZpositive cells in 9.0 dpc null embryos were located in the enlarged tail bud region and few, if any, were detected in the further rostral region (Fig. 2B). Cells in the enlarged tailbud of pMesogenin1 null animals also showed elevated apoptosis (Fig. 2I-L). There were few 14.5 dpc homozygous mutant fetuses, and those that survived lacked a tail and showed a grossly normal but very thin body with relatively normal forelimbs and hindlimbs (Fig. 2D). Taken together, the results indicate that cells that would normally form the presomitic paraxial mesoderm of the mouse have passed through the primitive streak but subsequently fail to mature normally, fail to form somites, and begin to self-destruct by apoptosis.

\section{Disregulated Notch signaling in pMesogenin $1^{-/-}$ embryos}

Failure to form morphologically detectable trunk/tail somites in pMesogenin1 homozygous mutant embryos points to the regulatory machinery that controls somitogenesis and segmentation. The Notch/Delta signaling system is known to be important for proper segmentation and successful somitogenesis (Gossler and de Angelis 1998; Jiang et al. 1998; Pourquie 1999; Dale and Pourquie 2000), although no single gene from this pathway has, upon knockout, produced a phenotype as severe as pMesogenin $1^{-/-}$. pMesogenin 1 null embryos showed disruption of multiple components of this system, and all deficits observed were confined to the presumptive paraxial mesoderm. Notch1 and 2 expression were both abolished in the paraxial mesoderm of null embryos at 9.5 dpc (Fig. 3A,B). Expression of the Notch ligand, D11-3 (Delta-like 3), was also undetectable in the homozygous mutant embryos (Fig. 3D). In contrast, expression of another Notch ligand, Dll-1 was not severely affected (Fig. 3C), although we noted what appeared to be a consistently reduced intensity in D11-1 in situ hybridizations in homozygotes.

Lunatic fringe and Hes1 are of particular interest because their RNA expression has been shown to oscillate in the PSM with periodicity linked to the period of new somite formation (Palmeirim et al. 1997; Forsberg et al. 1998; McGrew et al. 1998; Aulehla and Johnson 1999; Jouve et al. 2000). At the molecular level, they are thought to function in regulating and modulating Notch signaling. Both Lunatic fringe and Hes1 expression was abolished in the PSM of pMesogenin1 $9.5 \mathrm{dpc}$ null embryos (Fig. 3E,F). However, deficiency of Notch/Delta gene expression in pMesogenin1 null embryos is limited to the paraxial mesoderm domain, as Notch1, D11-1, and Lunatic fringe RNA expression in the neural tube and other neuronal cells was unaffected (Fig. 3A,C,E). Thus, Notch/Delta signaling components, including elements most intimately associated with the somitic clock, are disrupted in pMesogenin 1 homozygous mutant embryos in the domain of pMesogenin1 expression. The reverse, 

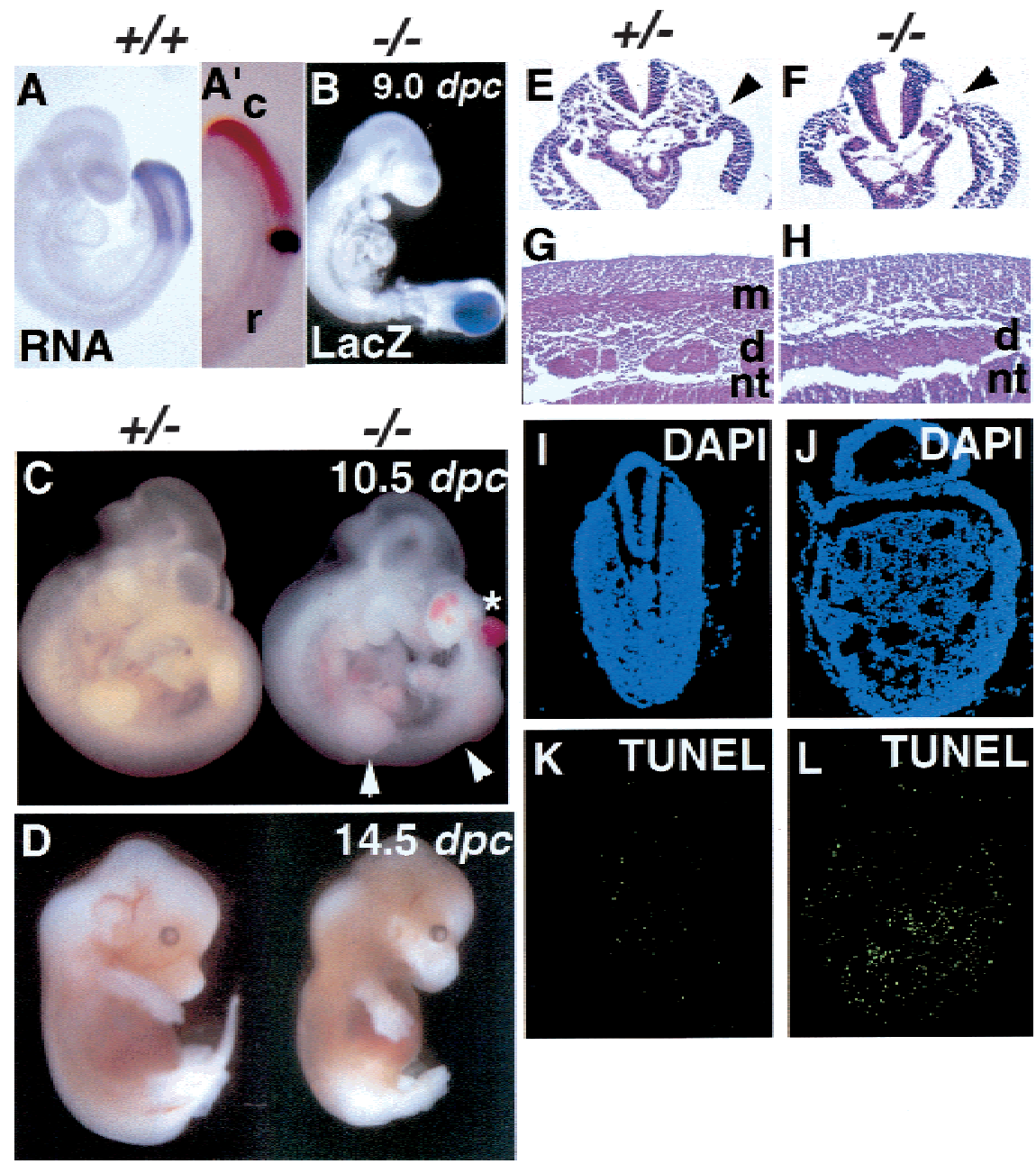

Figure 2. Disruption of pMesogenin1 results in defects of paraxial mesoderm formation. (A) Whole-mount in situ hybridization of pMesogenin1 RNA in wild type embryo of 9 dpc. $\left(\mathrm{A}^{\prime}\right)$. Both pMesogenin1 (reddish brown) and MesP2 (dark blue) RNAs were detected simultaneously in 9 dpc embryo by two-color whole-mount in situ hybridization. Expression of $p M e$ sogenin1 and MesP2 in PSM is mutually exclusive. This photo shows a lateral view of tailbud region. The rostral $(r)$ and caudal $(c)$ side of embryo is indicated. $(B)$ Whole-mount $\beta$-galactosidase histochemical staining in pMesogenin1 null embryo of $9 \mathrm{dpc}$ shows LacZ-positive cells are largely localized to the enlarged tailbud. (C) pMesogenin 1 null embryo of $10.5 \mathrm{dpc}$ shows no detectable segmented somites in the interlimb region indicated by two arrowheads. The null mutant also develops an enlarged tailbud and ectopic blood pools (asterisk). (D) At $14.5 \mathrm{dpc}$, the null mutant fetus lacks a tail (arrow), but possesses grossly normal limbs. $(E, F)$ Transverse sections of both control heterozygous $(E)$ and homozygous mutant embryos $(F)$ of $9.5 \mathrm{dpc}$ were stained with hematoxylin and eosin (H/E). Arrows indicate the boundary between paraxial mesoderm and lateral mesoderm. Paraxial mesoderm is absent in the null mutant $(F)$. $(G, H) \mathrm{H} / \mathrm{E}$ staining of parasagittal sections at interlimb region from $11.5 \mathrm{dpc}$ embryos also show lack of myotomes and presence of unsegmented DRG in homozygous mutant embryos $(H)$, but not in control heterozygous mutant embryos $(G)$. Abbreviations: d, dorsal root ganglia; m, myotome; and nt, neural tube. $(I-L)$ Increased apoptosis was observed in the tailbud of pMesogenin1 null embryos of $9.5 \mathrm{dpc}$. Cryosections of both control $(I, K)$ and null mutant embryos $(J, L)$ were subjected to TUNEL $(K, L)$ in the presence of fluorescein-conjugated dT and costained with DAPI $(I, J)$.

however, is not true, at least in one case. pMesogenin1 expression was not affected detectably in Notch1 null mutant embryos (Fig. 3H), which suggests two things about their regulatory relationship: pMesogenin1 likely acts upstream of Notch1, and feedback from Notch1 is not critical for pMesogenin1 expression. However, it is worth noting that the Notch1 null mutant gives a rather mild phenotype, perhaps because of redundancy with Notch2. Therefore, definitive conclusions about the relationship between pMesogenin 1 and possible upstream Notch/Delta signaling components await a more comprehensive study of Notch system mutants.

MesP1 and MesP2 are a pair of recently duplicated genes that are closely related to pMesogenin1 (Saga et al. 1996; Saga et al. 1997). Molecular phylogeny places the three genes together in a distinct bHLH subgroup (Yoon et al. 2000). Both are normally expressed in one or two stripes in the most rostral presomitic mesoderm /Saga et al. 1996; Saga et al. 1997; Sawada et al. 2000). Domains of MesP1/2 and pMesogenin1 expression abut each other quite closely but are strictly mutually exclusive (Fig. 2A, $\mathrm{A}^{\prime}$ )(Yoon et al. 2000). MesP2 homozygous mutant mice display a severe segmentation defect (although not as severe as pMesogenin1), which includes diminished Notch1 expression in the rostral domain of the presomitic mesoderm (Saga et al. 1997). MesP2 expression was abolished in pMesogenin1 homozygous mutant embryos at $9.5 \mathrm{dpc}$ when it would normally be prominent (Fig. 3G).

\section{Absence of differentiated derivatives of paraxial mesoderm in pMesogenin1 homozygous mutant animals}

The myogenic marker, Myf-5 at $10.0 \mathrm{dpc}$, was detected in somites of both heterozygotes and homozygotes (Fig. 4A). In null embryos, the most rostral somites had normal appearing Myf-5 expression, but the somites near the forelimbs had Myf-5 expression domains that become progressively smaller and more irregular in shape 
Figure 3. $(A-F)$ Molecular components of segmentation including the Notch/Delta pathway were severely disrupted in $p M e$ sogenin1 null mutants. Expression of Notch1 $(A)$, Notch $2(B)$, Dll-1 $(C)$, Dll-3 $(D)$, Lunatic fringe $(E)$, Hes $1(F)$, and MesP2 $(G)$ were examined in both heterozygous and homozygous mutant embryos of 9.5 dpc. $(H)$ Expression of pMesogenin1 in Notch1 null mutant embryos of 9 dpc. In panels $A$ to $H$, the heterozygous embryos are positioned at the left side and the homozygous embryo at the right side. The PSM of control embryos and the corresponding presumptive PSM in mutant embryos are marked by black brackets. In some cases, the head of the embryo has been removed to facilitate photography. In panel $A$, the rostral half of the trunk of the homozygous mutant embryo is in a twisted position. The consequence is that the dorsal side of the embryo at the mid trunk level faces outward toward the reader.
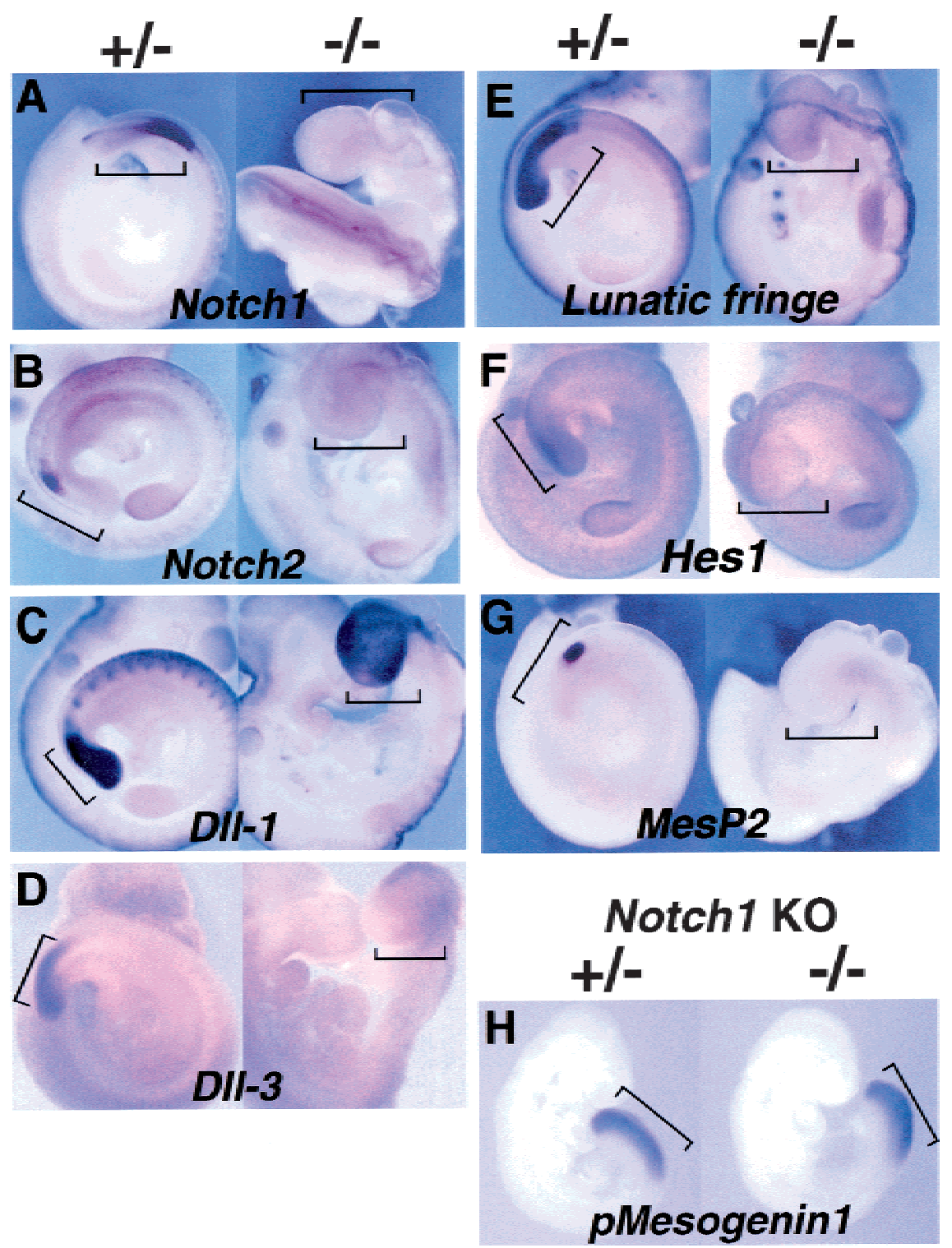

in a rostral to caudal gradient. Posterior to the forelimbs, Myf-5 was never detected in null embryos (Fig. 4A). Similarly, in older surviving embryos immunohistochemical assays detected no expression of markers of differentiated muscle, such as $\alpha$-actinin or myosin heavy chain (MHC)(data not shown) in the trunk region posterior to forelimbs (Fig. 5C-F). As with Myf-5, expression of $\alpha$-actinin at cervical and rostral forelimb levels appeared normal in the homozygous mutants (Fig. 5A,B).

Paraxis expression, which marks rostral presomitic mesoderm and developing somites (Burgess et al. 1995), was also defective in the posterior somites (11 and thereafter) and the presomitic mesoderm (Fig. 4B). Surviving fetuses at 14.5 and $17.5 \mathrm{dpc}$ displayed striking and informative skeletal defects (Fig. 6A-F). All vertebrae posterior to cervical level together with ribs were absent in homozygous mutants (Fig. 6A-F). Consistent with the division in phenotype between somites 1-11 and all more caudal ones, all cervical vertebrae were present, although the most posterior of these were sometimes malformed and fused (Fig. 6E,F). The latter observation suggests that the requirement for pMesogenin 1 begins gradually, implying a role for pMesogenin1 in regulating formation of at least some cervical vertebrae as well. Other trunk skeletal structures that originate from lateral mesoderm, such as the sternum and scapula, formed quite normally. We conclude that trunk skeletal and muscle defects in the mutant are autonomous to paraxial mesoderm derivatives.

Because pMesogenin1 homozygous mutant embryos are devoid of detectable paraxial mesoderm at the hindlimb level at the time hindlimb muscle progenitors would normally migrate into the developing hindlimb, a simple prediction is that no muscle precursors will enter the hindlimb bud and that hindlimbs will develop without muscle. Conversely, forelimbs might be expected to 

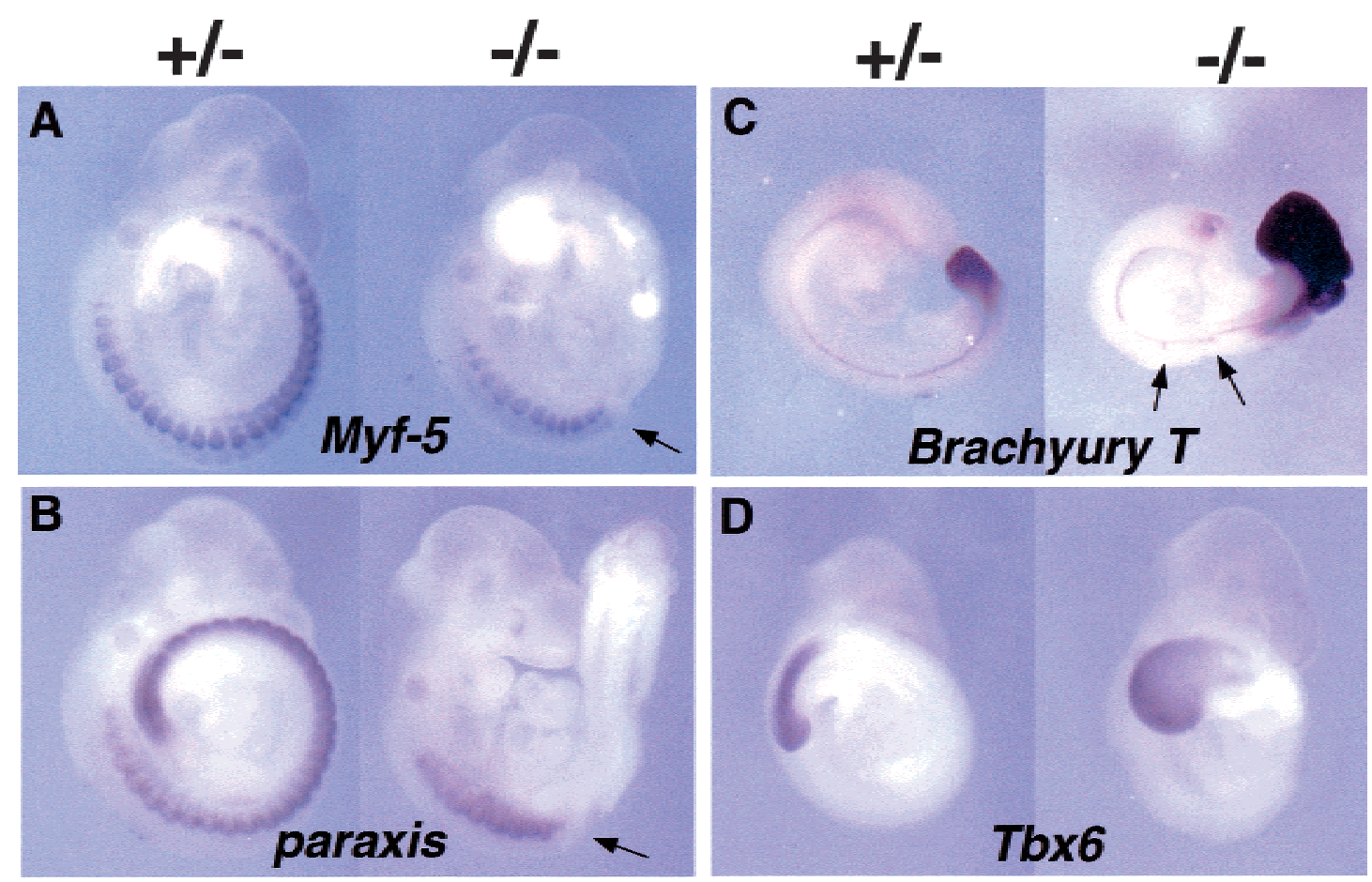

Figure 4. Whole-mount in situ analysis of gene expression. In each panel, the control heterozygous embryo is located on the left and the null mutant embryo on the right. (A) Early myogenic marker Myf-5 expression in mutant embryo was normal in rostral somites, but was not detected in the trunk domain posterior to the forelimbs, where somites are normally present. The arrow indicates the boundary between $M y f-5$ positive and negative domains. (B) Paraxis expression was also affected in the domain posterior to forelimbs in the pMesogenin1 homozygous mutant embryo. The arrow represents the boundary between paraxis positive and negative domains. (C) Brachyury $T$ expression in caudal paraxial mesoderm was upregulated in the null embryos of 9.5 dpc, but the expression in notochord was grossly unaffected. Arrows indicate the Brachyury T staining area where the notochord is kinked. $(D)$ The expression of Tbx6 was not affected in the 9.5 dpc pMesogenin1 null mutants.

develop significant skeletal muscle, as somites (numbers 9 to 13) are known to contribute to the forelimb muscle (Richardson et al. 1998) and are present, albeit somewhat reduced in size, in the homozygous mutant embryos. These predictions fit the observed phenotype precisely. As measured by expression of $\alpha$-actinin, muscle was absent from hindlimbs of null embryos at $12.5 \mathrm{dpc}$, whereas its expression in forelimbs was grossly normal (data not shown). This agrees well with the presence of Pax3 positive muscle precursors entering the forelimb buds but not detected in the hindlimb buds at earlier developmental times (data not shown).

\section{Negative regulation of Brachyury $\mathrm{T}$}

The murine T-box transcriptional regulators, Brachyury $T$ and $T b x 6$, are known to play major roles in mesoderm development (Smith 1999). We observed that Brachyury $T$ RNA is expressed in the presumptive paraxial mesoderm of pMesogenin1 homozygous mutant embryos at levels consistently higher than those of heterozygous or wild type littermates (Fig. 4C). In the notochord where pMesogenin1 is never expressed, Brachyury T RNA was unaffected, as was another notochord marker, shh (data not shown), indicating that notochord formation and dif- ferentiation in homozygous mutant embryos is largely normal by both molecular and anatomic criteria (Fig. $2 \mathrm{~F}, \mathrm{H})$. This result implies that $p M e s o g e n i n 1$ normally has a negative regulatory effect on Brachyury $T$ levels in the PSM, although it is not known whether this effect is direct or indirect. In contrast $T b \times 6$ expression was relatively unaffected (Fig. 4D). Tbx6 is of particular interest because its disruption causes paraxial mesoderm tissue to assume a neural fate /Chapman and Papaioannou 1998). This has led to the proposition that it is the major specification gene for paraxial mesoderm. Also, unlike any other gene known to us, the Tbx6 RNA is expressed in a spatiotemporal pattern that is virtually identical to that of pMesogenin1 (Chapman et al. 1996; Yoon et al. 2000). We infer from this result that pMesogenin1 is not an upstream regulator of $T b \times 6$. Similarly, in preliminary experiments, pMesogenin1 expression was not detectably changed in Tbx6 null mutant embryos (Yoon, Papaionnou, and Wold, data not shown). This argues that Tbx6 is not an upstream regulator of pMesogenin1 and suggests that these regulators likely operate in parallel.

\section{Discussion}

Our results show that in the mouse pMesogenin 1 is essential for proper maturation and segmentation of pos- 

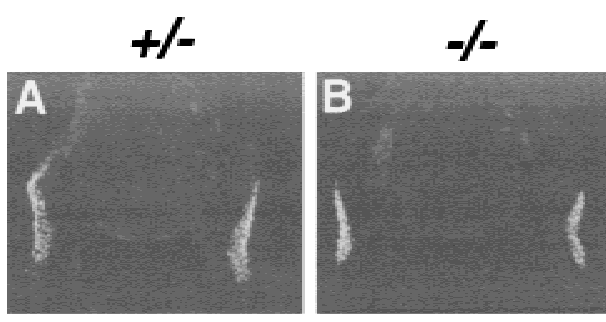

Cervical
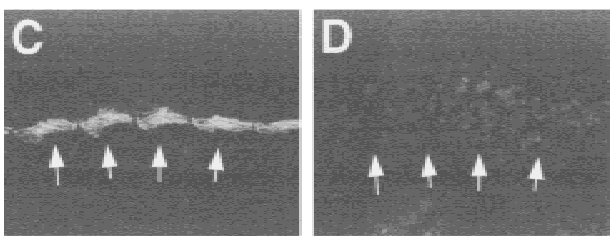

Interlimb
level
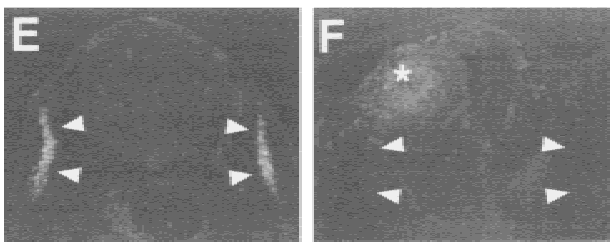

Hindlimb level

Figure 5. Absence of differentiated muscles in pMesogenin 1 null mutants. $(A-F)$ Expression of differentiated skeletal muscle marker $\alpha$-actinin. Immunofluorescent staining of anti $\alpha$-actinin antibodies on transverse sections at cervical level $(A, B)$ and hindlimb level $(E, F)$, and parasagittal section of interlimb region $(C, D)$ of $11.5 \mathrm{dpc}$ embryos. Myotomal staining of $\alpha$-actinin in the interlimb and the hindlimb region was absent in the homozygous mutant embryos $(D, F)$, whereas expression of $\alpha$-actinin at the cervical level was unaffected in the null embryo $(A, B)$. Arrows (panels $C, D$ ) and arrowheads (panels $E, F$ ) indicate the position of myotomes (panels $C, E$ ) in the control animals and the corresponding location in the homozygous mutants (panels $D, F)$. An asterisk in panel $F$ indicates a weak background staining from blood cells found in the ectopic blood pool.

terior paraxial mesoderm. The earliest and most obvious morphological defect in pMesogenin1 null embryos is a failure of somitogenesis and the formation of an enlarged tailbud. Later in development there is a striking absence of all trunk and tail skeletal muscle and axial skeleton, whereas the hindlimb skeleton, derived from lateral mesoderm, was spared. Cells of the unsegmented presumptive paraxial mesoderm of homozygous mutant embryos appear to be developmentally arrested. They do not progress to the stage of expressing the rostral-most PSM specific marker genes such as MesP1/2, nor do they express paraxis, Myf-5, or other muscle marker genes that would normally be induced after somite formation. Elevated apoptosis in the tailbud domain and absence of paraxial cells in the interlimb region of older mutant embryos suggest a possible trophic requirement for pMesogenin1 and one or more of its regulatory targets.

Notch/Delta signaling apparatus and oscillating somitic clock genes depend on pMesogenin 1

A key conclusion of this work is that pMesogenin1 is an essential upstream regulator of multiple members of the
Notch/Delta signaling apparatus, which is known to be important for proper somitogenesis and segmentation

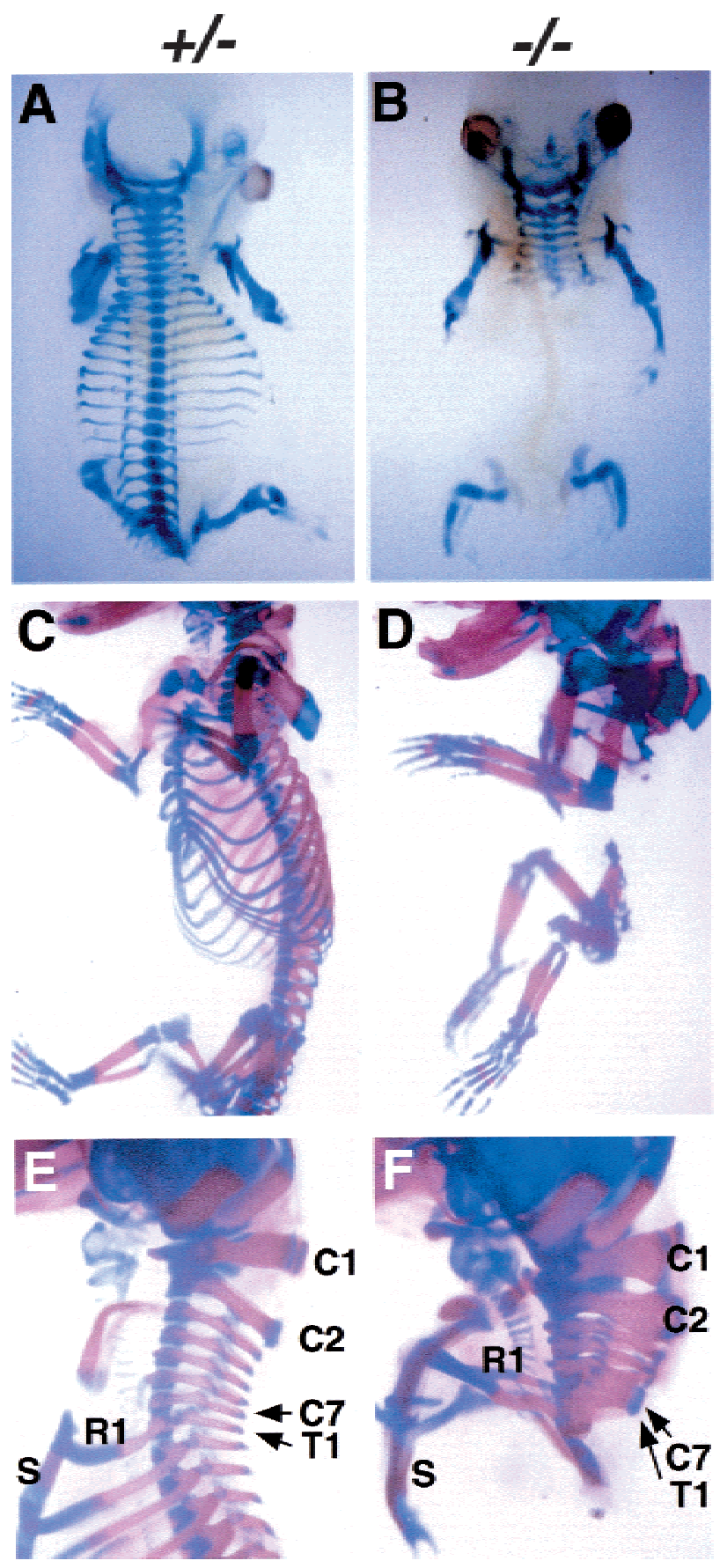

Figure 6. $(A-F)$ Whole-mount skeleton preparation of heterozygous $(A, C, E)$ and homozygous mutants $(B, D, F)$ of $14.5 \mathrm{dpc}$ $(A, B)$ and $17.5 \mathrm{dpc}(C-F)$. Bones and cartilage stained red and blue by Alizarin Red S and Alcian Blue, respectively. All of the trunk skeleton posterior to the forelimbs (thoracic to downward) was missing in the null mutants $(B, D, F)$, whereas head skeleton, cervical vertebrae, and limb skeleton were largely normal. Some cervical vertebrae in the null mutants were fused and malformed, indicating a milder defect than that observed in the trunk and tail domains. Abbreviations: Cn, cervical vertebrae \#n; R1, rib \#1; S, sternum; and T1, thoracic vertebrae \#1. 
(Pourquie 1999; Dale and Pourquie 2000). This epistasis relationship is tissue autonomous, being entirely restricted to the presumptive PSM of the trunk and tail where pMesogenin 1 is normally expressed. Thus $p M e$ sogenin1 is absolutely required for expression of Notch1, Notch2, D11-3, Hes1, and Lunatic fringe in the trunk and tail PSM, whereas D11-1 appeared slightly reduced in RNA expression in the absence of pMesogenin1 (Fig. 3). The intensity of the somitogenesis/segmentation defect is greater in this mutant than in any of the individual Notch/Delta gene knockouts, perhaps because it affects more components of the system simultaneously.

The precise role or roles of Notch signaling in somitogenesis is presently unclear, although it appears to be linked to the recently discovered molecular clock that operates in the same domain of the PSM (Pourquie 1999; Dale and Pourquie 2000). Genes such as Lunatic fringe and Hes1 display RNA expression patterns in the tail bud that oscillate with the same periodicity as the somite formation that they anticipate (Palmeirim et al. 1997; Forsberg et al. 1998; McGrew et al. 1998; Aulehla and Johnson 1999; Nakagawa et al. 1999; Jouve et al. 2000). These genes have thereby revealed the existence of a somitic clock (Pourquie 1999; Dale and Pourquie 2000), and gene knockout studies in mice have shown they are also required for proper somitogenesis (Evrard et al. 1998; Zhang and Gridley 1998). A second conclusion from this work is that pMesogenin1 is absolutely required for expression of these oscillating genes. The deficit in pMesogenin 1 homozygous mutant embryos was not limited to failure of this RNA to oscillate, but instead caused absence of any detectable Lunatic fringe and Hes1 transcripts (Fig. 3E,F). The molecular mechanism by which pMesogenin1 ultimately acts as a positive regulator of Notch- and clock-regulated genes is unknown, and could be an indirect one. However, because pMesogenin 1 is a bHLH class protein, an obvious and simple possibility is that it might be a direct transcriptional regulator of one or more of these genes.

\section{Relationship of pMesogenin1 with MesP1/2}

MesP1/2 are by far the most closely related paralogs of pMesogenin1 (Yoon et al. 2000). Phylogenetic analysis showed that MesP1/2 are the most closely related paralogs of pMesogenin1 based on bHLH domain protein sequences (Yoon et al. 2000). MesP2 is normally expressed in the PSM, but it is restricted to a transient anterior stripe that forms just before overt somite formation (Fig. 2A') (Saga et al. 1996; Saga et al. 1997). As somitogenesis proceeds caudally, MesP2 RNA appears shortly after pMesogenin 1 expression ceases, and this mutually exclusive pattern of expression in the PSM extends to their Xenopus and chicken orthologs (Buchberger et al. 1998; Sparrow et al. 1998; Yoon et al. 2000). MesP2 null mouse embryos are also defective in somitogenesis and segmentation, and at the molecular level Notch signaling pathway genes are again dramatically affected (Saga et al. 1997). In Xenopus and zebrafish, there is a similar picture in which the candidate orthologs of MesP1/2, Thyl- acine 1 and 2 (Xenopus), and mesp- $a$ and $b$ (zebrafish) are expressed in stripes in the rostral PSM (Sparrow et al. 1998; Sawada et al. 2000). The initial functional analysis suggests mesp- $b$ of zebrafish is important for correct segmentation and for defining anterior versus posterior compartments of the somite (Sawada et al. 2000). Similarly, Thylacine1/2 in Xenopus act on segmentation in conjunction with Notch signaling (Sparrow et al. 1998). Thus, it appears that all three genes (pMesogenin1, MesP1, and 2) share the property of regulating expression of Notch family members, but that they do so in different spatiotemporal domains of the PSM.

Other possible roles for pMesogenin 1 in specification, cell survival, or gastrulation

Because the phenotype of the pMesogenin1 null mutants is so robust with respect to known molecular markers in the PSM, it is possible that these cells are developmentally arrested in a state that normally precedes the onset of expression of any segmentation or clock genes. Thus, pMesogenin1 might operate primarily as a regulator of paraxial mesoderm specification, and secondarily as a regulator of segmentation and somitogenesis. There is some support for this idea of a broader and perhaps developmentally earlier effect from gain-of-function experiments done previously with the Xenopus ortholog of pMesogenin1 (Yoon et al. 2000). In that system, we found that ectopic Xenopus pMesogenin1 expressed in Xenopus embryo animal cap explants, which would otherwise assume only ectodermal fates, induced the expression of $X w n t 8, X M y o D$, and $X M y f-5$ which are first expressed before formation of tailbud paraxial mesoderm. In addition, genes involved in segmentation of the tailbud (ESR4/5 of the Hairy/Enhancer of Split family) were also induced (Yoon et al. 2000). Xwnt8 is known to be a powerful inducer of ventrolateral mesoderm phenotype. Unfortunately, a convincing candidate ortholog for Tbx6 in Xenopus has not yet been identified. However, our preliminary experiments with $T b x 6$ mutant mice, in which pMesogenin 1 continued to be expressed in the tailbud domain, suggest that pMesogenin1 alone cannot specify the complete paraxial mesoderm phenotype, as those cells apparently went on to convert to a neuroectodermal fate. Conversely, Tbx6 continued to be expressed in pMesogenin1 null mutants (Fig. 4D). Thus, the two genes appear to be regulated independently of each other within nearly identical expression domains, which argues that both may contribute to specification.

Another formal possibility is that the first effect of pMesogenin1 is on cell migration during gastrulation, which then affects all subsequent events. There is evidence for this in the FGFR1 pathway (Ciruna et al. 1997; Deng et al. 1997; Saxton and Pawson 1999) and for Brachyury T (Wilson et al. 1995). In those mutants, however, the gastrulation defects were not restricted to paraxial mesoderm, but instead displayed significant additional effects on axial and lateral mesoderm. A distinction in pMesogenin1 homozygous mutant embryos is that both lateral mesoderm, as represented by the ster- 
num, scapula and the hindlimb skeletons, and axial mesoderm (notochord) were remarkably normal (Figs. 4C, 6). This argues that migration of mesodermal cells is not globally defective, even at the position of the future hindlimb, which is much affected in pMesogenin1 homozygous mutant embryos, but it leaves open the possibility of a migratory defect that is specific and autonomous to presumptive paraxial mesoderm. A chimeric embryo study will be needed to clarify this issue.

Interestingly, presomitic mesodermal cells in the mutant also showed elevated apoptosis (Fig. 2I-L), and this may explain the greatly reduced cell mass between forelimb and hindlimb levels in day 9.5 and older embryos. A simple interpretation is that pMesogenin1 serves a trophic function for cells of the tailbud domain, and subsequent developmental events are thwarted because cell death precedes or is triggered by the signals that normally initiate segmentation and/or somite patterning. The fact that pMesogenin 1 is expressed in the tailbud of Tbx6 null animals may contribute to the ongoing survival of cells that convert to ectopic neural tubes in the position where paraxial mesoderm would normally be.

\section{The mystery of head/neck versus trunk/tail paraxial mesoderm}

With respect to the pMesogenin1 mutant phenotype, head and neck somites are dramatically different from trunk and tail domain somites. Somites above the forelimb are quite normal in pMesogenin1 homozygous mutant embryos, whereas somites nearing the forelimb level are progressively smaller and more malformed, and all somites below forelimb level are greatly affected (Fig. $4 \mathrm{~A}, \mathrm{~B})$. What does this mean for understanding $p M e$ sogenin1 function, or more broadly, for understanding general principles of somitogenesis and segmentation in vertebrates? First, it is noteworthy that this curious distinction between head/neck and trunk/tail domains is not unique to pMesogenin 1 mutants. In fact, the distinction seems relatively common. For example, trunk paraxial mesoderm posterior to the forelimbs in Brachyury $T$ and Wnt3a null mutants is severely affected, whereas the anterior domain is apparently normal (Beddington et al. 1992; Takada et al. 1994). Similarly, mutation of the pMesogenin 1 family bHLH gene MesP2 also eliminated proper trunk and tail domain somitogenesis whereas the head and neck domain somites formed normally (Saga et

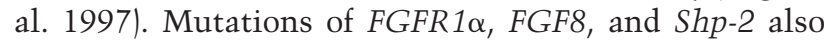
affect segmentation and somitogenesis and the effects have been described as weak or undetectable in the head and neck but are evident below the forelimb level (Saxton et al. 1997; Sun et al. 1999; Xu et al. 1999). This is even the case for $T b \times 6$, which is viewed as a strong paraxial mesoderm determination gene (Chapman and Papaioannou 1998). Thus, there is persuasive evidence for the proposition of Chapman and coworkers (1998) that Tbx6 is required to specify a paraxial mesodermal fate, with the alternative being a default to a neuroectodermal fate in the trunk and tail. This leaves unanswered the question of what gene or genes direct the same event in the head and neck domain.

The consistent underlying theme, which varies in intensity from one gene to another and one allele to another, is that the head/neck domain of paraxial mesoderm is, in some rather profound way, different from the remainder of the paraxial mesoderm with respect to regulation of specification and segmentation. An explanation with interesting evolutionary implications is that head and neck paraxial mesoderm employs a distinct set of undiscovered or uncharacterized genes to regulate specification and segmentation in a manner similar to the better studied and more experimentally accessible trunk and tail domains. For pMesogenin1, we can begin to probe this possibility by asking whether pMesogenin 1 is expressed in progenitors of the most rostral paraxial mesoderm. If it is selectively expressed in progenitors of trunk and tail domains alone, the argument for a different mechanism or regulation by different genes would be greatly strengthened. We have shown that pMesogenin1 RNA is detected early enough (day 7) and in a spatial domain (presumptive mesoderm just outside the primitive streak) so that progenitor cells of the first 11 somites might be included (Yoon et al. 2000). However, we cannot definitively conclude that these cells include all (or even any) progenitors of the first dozen somites that comprise the head and neck (Yoon et al. 2000). The presence of some malformed and fused cervical vertebrae in the pMesogenin 1 homozygous mutants seems to imply relevant expression of pMesogenin 1 in at least a portion of progenitor cells of head and neck somites (Fig. 6F). Based on its expression pattern, a similar question about the extent and cellularity of expression in the head and neck domain could hold for Tbx6. For both genes, Crerecombinase mediated lineage tracing studies (Zinyk et al. 1998) in which Cre protein is expressed in the pattern of pMesogenin 1 or Tbx6 in the presence of a recombinase dependent reporter gene (Soriano 1999) should resolve whether these regulators are restricted to progenitors of trunk and tail paraxial mesoderm, or are also active in precursors of the head and neck somites.

\section{Materials and methods}

Generation of pMesogenin 1 mutant mouse strains

Genomic DNA for mouse pMesogenin1 was isolated from $\lambda$ phage 129SvJ genomic DNA library (Stratagene) using mouse full-length $p M e s o g e n i n 1 \mathrm{cDNA}$ as a probe. The targeting vector (Fig. 1A) was transfected into CJ7 ES cells and homologous recombinants were identified by Southern hybridization. Blastocyst injection and chimera production was done in the Caltech Transgenic Facility. Two independent mutant lines

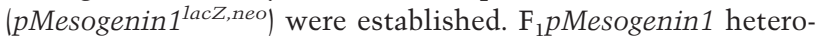
zygous males and females were obtained from the matings between founder of the heterozygous male (129SvJ background) and C57BL6 wild type females. These animals were intercrossed to collect homozygous mutants and heterozygous littermate controls. We found that the developmental phenotypes of homozygous mutants from the two founders were indistinguishable. Subsequently, the two founding strains were used inter- 
changeably in the experiments reported. A pMesogenin $1^{\text {lacz }}$ allele in which the PGK neo cassette was deleted was generated by crossing the pMesogenin $1^{\text {lacz,neo }}$ heterozygous mice to CMV-Cre transgenic mice (from the Caltech Transgenic Facility). Deletion of the neo gene was verified by PCR. The anatomic and developmental phenotypes of pMesogenin1lacZ homozygous embryos were indistinguishable from those of pMesogenin $1^{1 a c z, \text { neo }}$ homozygous animals. We conclude that in this knock-out/knock-in, the presence of the neomycin selection cassette does not significantly affect phenotypes.

\section{Embryo collection and genotyping}

Mouse embryos were collected from matings between the heterozygous animals and staged by counting the noon of day of plug as $0.5 \mathrm{dpc}$. Yolk sac DNA was extracted from individual embryos and subjected to PCR genotyping. For pMesogenin1 null embryos, P1 (5'-CCAAGGAGCCTTGTACTGCTGC-3'), P2 (5'-GCCACCAGCAGTGTGTAGATAGGGAGGT-3'), and Z (5'-GCAAAGCGCCATTCGCCATTC-3') primers were used. The presence or absence of the neo gene was determined by PCR using N1 (5'-GATCGGCCTTGAACAAGATGGATTGCA-3') and N2 (5'-AGCTCTTCAGCAATATCACGGGTAGCCA-3') primers (the sequences were provided by Dr. Jae Sang Kim). Notch1KO mice were obtained from the Jackson Laboratory, and the genotypes of Notch1 null embryos were analyzed by PCR as described earlier (Conlon et al. 1995).

\section{Analysis of pMesogenin 1 null phenotypes}

Paraffin sections of embryos were prepared and stained with hematoxylin and eosin $(\mathrm{H} / \mathrm{E})$ as described elsewhere (Patapoutian et al. 1995). 10-14 $\mu \mathrm{m}$ frozen sections of OCT-embedded mouse embryo were stained with anti $\alpha$-actinin antibodies (Sigma, $1: 400$ ), followed by incubation with FITC-conjugated Donkey anti mouse IgG antibodies (Jackson Immuno Research) as described previously (Patapoutian et al. 1995). TUNEL staining on cryosections was done using a cell death detection kit (Boehringer Mannheim). Whole-mount in situ hybridization was performed according to the protocol previously described (Yoon et al. 2000). Skeletons of fetuses were stained with Alizarin Red S for bone and Alcian blue for cartilage as described elsewhere (Patapoutian et al. 1995).

\section{Acknowledgments}

We thank Drs. Rosa Beddington, Ron Conlon, Sean Egan, Achim Gossler, Tom Gridley, Chee Gun Lee, Ryoichiro Kageyama, Eric Olson, and Virginia Papaioannou for providing their valuable reagents and protocols; Drs. David Anderson, Marie Ceste, Jae Sang Kim, Randall Moon, and Brian Williams for critically reading the manuscript; and Shirley Pease, Shuling Wang, and Xin Yu for their excellent technical assistance. This work was supported by grants from NIH NIAMS to B.J.W.

The publication costs of this article were defrayed in part by payment of page charges. This article must therefore be hereby marked "advertisement" in accordance with 18 USC section 1734 solely to indicate this fact.

\section{References}

Aulehla, A. and Johnson, R.L. 1999. Dynamic expression of lunatic fringe suggests a link between Notch signaling and an autonomous cellular oscillator driving somite segmentation. Dev. Biol. 207: 49-61.
Beddington, R.S.P., Rashbass, P., and Wilson, V. 1992. Brachyury - a gene affecting mouse gastrulation and early organogenesis. Development (suppl.): 157-165.

Buchberger, A., Seidl, K., Klein, C., Eberhardt, H., and Arnold, H.H. 1998. cMeso-1, a novel bHLH transcription factor, is involved in somite formation in chicken embryos. Dev. Biol. 199: 201-215.

Burgess, R., Cserjesi, P., Ligon, K.L., and Olson, E.N. 1995. Paraxis - a basic helix-loop-helix protein expressed in paraxial mesoderm and developing somites. Dev. Biol. 168: 296-306.

Chapman, D.L. and Papaioannou, V.E. 1998. Three neural tubes in mouse embryos with mutations in the T-box gene Tbx6. Nature 391: 695-697.

Chapman, D.L., Agulnik, I., Hancock, S., Silver, L.M., and Papaioannou, V.E. 1996. Tbx6, a mouse T-box gene implicated in paraxial mesoderm formation at gastrulation. Dev. Biol. 180: $534-542$.

Ciruna, B.G., Schwartz, L., Harpal, K., Yamaguchi, T.P., and Rossant, J. 1997. Chimeric analysis of fibroblast growth factor receptor-1 (Fgfr1) function: A role for FGFR1 in morphogenetic movement through the primitive streak. Development 124: 2829-2841.

Conlon, R.A., Reaume, A.G., and Rossant, J. 1995. Notch1 is required for the coordinate segmentation of somites. Development 121: 1533-1545.

Dale, K.J. and Pourquie, O. 2000. A clock-work somite. Bioessays 22: $72-83$.

deAngelis, M.H., McIntyre, J., and Gossler, A. 1997. Maintenance of somite borders in mice requires the Delta homologue DIIl. Nature 386: 717-721.

Deng, C.X., Bedford, M., Li, C.L., Xu, X.L., Yang, X., Dunmore, J., and Leder, P. 1997. Fibroblast growth factor receptor-1 (FGFR-1) is essential for normal neural tube and limb development. Dev. Biol. 185: 42-54.

Evrard, Y.A., Lun, Y., Aulehla, A., Gan, L., and Johnson, R.L. 1998. Lunatic fringe is an essential mediator of somite segmentation and patterning. Nature 394: 377-381.

Forsberg, H., Crozet, F., and Brown, N.A. 1998. Waves of mouse Lunatic fringe expression, in four-hour cycles at two hour intervals, precede somite boundary formation. Curr. Biol. 8: $1027-1030$

Gossler, A. and de Angelis, M.H. 1998. Somitogenesis. In Current Topics in Developmental Biology Vol 38, pp. 225-287.

Jen, W.C., Wettstein, D., Turner, D., Chitnis, A., and Kintner, C. 1997. The Notch ligand, X-Delta-2, mediates segmentation of the paraxial mesoderm in Xenopus embryos. Development 124: 1169-1178.

Jen, W.C., Gawantka, V., Pollet, N., Niehrs, C., and Kintner, C. 1999. Periodic repression of Notch pathway genes governs the segmentation of Xenopus embryos. Genes \& Dev. 13: 1486-1499.

Jiang, Y.J., Smithers, L., and Lewis, J. 1998. The clock is linked to Notch signalling. Curr. Biol. 8: 868-871.

Joseph, E.M. and Cassetta, L.A. 1999. Mespo: A novel basic helix-loop-helix gene expressed in the presomitic mesoderm and posterior tailbud of Xenopus embryos. Mech. Dev. 82: 191-194.

Jouve, C., Palmeirim, I., Henrique, D., Beckers, J., Gossler, A., Ish-Horowicz, D., and Pourquie, O. 2000. Notch signalling is required for cyclic expression of the hairy-like gene Hes1 in the presomitic mesoderm. Development 127: 1421-1429.

Kusumi, K., Sun, E.S., Kerrebrock, A.W., Bronson, R.T., Chi, D.C., Bulotsky, M.S., Spencer, J.B., Birren, B.W., Frankel, W.N., and Lander, E.S. 1998. The mouse pudgy mutation disrupts Delta homologue D1l3 and initiation of early somite 
boundaries. Nat. Genet. 19: 274-278.

McGrew, M.J., Kim Dale, J., Fraboulet, S., and Pourquie, O. 1998. The lunatic Fringe gene is a target of the molecular clock linked to somite segmentation. Curr. Biol. 8: 979-982.

Nakagawa, O., Nakagawa, M., Richardson, J.A., Olson, E.N., and Srivastava, D. 1999. HRT1, HRT2, and HRT3: A new subclass of bHLH transcription factors marking specific cardiac, somitic, and pharyngeal arch segments. Dev. Biol. 216: $72-84$.

Oka, C., Nakano, T., Wakeham, A., Delapompa, J.L., Mori, C., Sakai, T., Okazaki, S., Kawaichi, M., Shiota, K., Mak, T.W., et al. 1995. Disruption of the mouse RBP-J-Kappa gene results in early embryonic death. Development 121:32913301.

Palmeirim, I., Henrique, D., IshHorowicz, D., and Pourquie, O. 1997. Avian hairy gene expression identifies a molecular clock linked to vertebrate segmentation and somitogenesis. Cell 91: 639-648.

Patapoutian, A., Yoon, J.K., Miner, J.H., Wang, S.L., Stark, K., and Wold, B. 1995. Disruption of the mouse MRF4 gene identifies multiple waves of myogenesis in the myotome. Development 121: 3347-3358.

Pourquie, O. 1999. Notch around the clock. Curr. Opin. Genet. Dev. 9: 559-565.

Richardson, M.K., Allen, S.P., Wright, G.M., Raynaud, A., and Hanken, J. 1998. Somite number and vertebrate evolution. Development 125: 151-160.

Saga, Y., Hata, N., Kobayashi, S., Magnuson, T., Seldin, M.F., and Taketo, M.M. 1996. MesP1: A novel basic helix-loophelix protein expressed in the nascent mesodermal cells during mouse gastrulation. Development 122: 2769-2778.

Saga, Y., Hata, N., Koseki, H., and Taketo, M.M. 1997. MesP2: A novel mouse gene expressed in the presegmented mesoderm and essential for segmentation initiation. Genes \& Dev. 11: 1827-1839.

Sawada, A., Fritz, A., Jiang, Y.J., Yamamoto, A., Yamasu, K., Kuroiwa, A., Saga, Y., and Takeda, H. 2000. Zebrafish Mesp family genes, mesp-a and mesp-b are segmentally expressed in the presomitic mesoderm, and mesp-b confers the anterior identity to the developing somites. Development 127: 16911702.

Saxton, T.M. and Pawson, T. 1999. Morphogenetic movements at gastrulation require the $\mathrm{SH} 2$ tyrosine phosphatase Shp2. Proc. Natl. Acad. Sci. USA 96: 3790-3795.

Saxton, T.M., Henkemeyer, M., Gasca, S., Shen, R., Rossi, D.J., Shalaby, F., Feng, G.S., and Pawson, T. 1997. Abnormal mesoderm patterning in mouse embryos mutant for the $\mathrm{SH} 2$ tyrosine phosphatase Shp-2. EMBO J. 16: 2352-2364.

Smith, J. 1999. T-box genes - what they do and how they do it. Trends Genet. 15: 154-158.

Soriano, P. 1999. Generalized lacZ expression with the ROSA26 Cre reporter strain. Nat. Genet. 21: 70-71.

Sparrow, D.B., Jen, W.C., Kotecha, S., Towers, N., Kintner, C., and Mohun, T.J. 1998. Thylacine 1 is expressed segmentally within the paraxial mesoderm of the Xenopus embryo and interacts with the Notch pathway. Development 125: 20412051.

Sun, X., Meyers, E.N., Lewandoski, M., and Martin, G.R. 1999. Targeted disruption of Fgf8 causes failure of cell migration in the gastrulating mouse embryo. Genes \& Dev. 13: $1834-1846$.

Swiatek, P.J., Lindsell, C.E., Franco del Amo, F., Weinmaster, G., and Gridley, T. 1994. Notch1 is essential for postimplantation development in mice. Genes \& Dev. 8: 707-719.

Takada, S., Stark, K., Shea, M.J., Vassileva, G., McMahon, J.A., and McMahon, A.P. 1994. Wnt-3a regulates somite and tail- bud formation in the mouse embryo. Genes \& Dev. 8: 174189.

Wilson, V., Manson, L., Skarnes, W.C., and Beddington, R.S.P. 1995. The T-Gene Is Necessary For Normal Mesodermal Morphogenetic Cell Movements During Gastrulation. Development 121: 877-886.

Xu, X.L., Li, C.L., Takahashi, K., Slavkin, H.C., Shum, L., and Deng, C.X. 1999. Murine fibroblast growth factor receptor 1 alpha isoforms mediate node regression and are essential for posterior mesoderm development. Dev. Biol. 208: 293-306.

Yoon, J.K., Moon, R.T., and Wold, B. 2000. The bHLH class protein pMesogenin 1 can specify paraxial mesoderm phenotypes. Dev. Biol. 222: 376-391.

Zhang, N. and Gridley, T. 1998. Defects in somite formation in lunatic fringe-deficient mice. Nature 394: 374-377.

Zinyk, D.L., Mercer, E.H., Harris, E., Anderson, D.J., and Joyner, A.L. 1998. Fate mapping of the mouse midbrain-hindbrain constriction using a site-specific recombination system. Curr. Biol. 8: 665-668. 


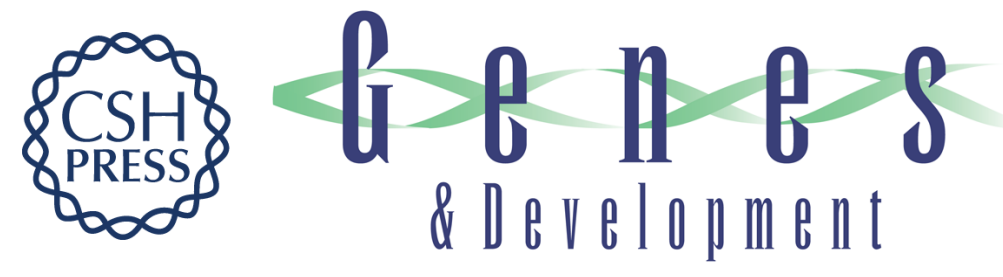

\section{The bHLH regulator pMesogenin1 is required for maturation and segmentation of paraxial mesoderm}

Jeong Kyo Yoon and Barbara Wold

Genes Dev. 2000, 14:

Access the most recent version at doi:10.1101/gad.850000

References This article cites 40 articles, 18 of which can be accessed free at: http://genesdev.cshlp.org/content/14/24/3204.full.html\#ref-list-1

License

Email Alerting Receive free email alerts when new articles cite this article - sign up in the box at the top Service right corner of the article or click here.

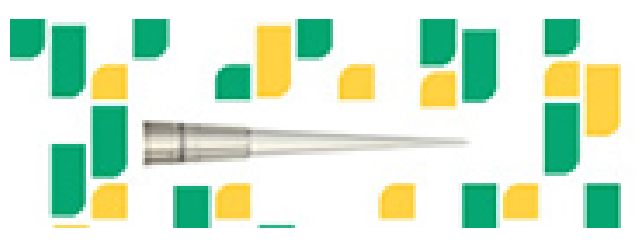

Focused on your science. 